\title{
And the journey begins...
}

Prof. Dr. Madhab Lamsal

The explosion of knowledge has increased unlimitedly with the rapid expansion of the information technology. Now even the "common man" has access to this vast pool of knowledge and resources with several apps at their fingertips. However, there is a grave challenge in quality control and accessing the correct information from the vast pool of this bewildering knowledge. Research be it positive or negative needs to be published, else it will be perished, incurring a great loss to the global society. Publication has become easier these days, however, the challenges of publishing in reputed journals are still persisting.

Similarly, research is being done in every field and health sector is no exception. Any findings, even if apparently felt of less importance, paradoxically may be of greater value, depending on the community and the field concerned. The emergence and reemergence of newer and older diseases pose yet another challenge. Thus, the research should find its place for publication in the right time in the right forum and should benefit people in general.

Clinical biochemistry is not restricted only to the diagnostic tools for metabolites, but can also be linked to molecular biology, proteomics, genomics and metabolomics, besides bioinformatics. It should find a rightful share also in therapeutics and individualized evidence based medicine.

Point of care testing has revolutionised the field of medical science with ample opportunities but creates a great challenge related to the quality services. Due to simplicity of operation, it may at times runs into the risk of displacing the role of qualified professionals. For example, the wide range of POCT devices in emergency setting may undermine the roles of biochemists in result interpretation as well as in overall patient management. Machines are intelligent but they cannot displace the human brain.

The machine equipment and reagent vendors should also be bound with high professional ethics so that the quality on the human health should not be compromised and jeopardised. The results obtained through laboratory investigation will be giving the quality not more than the input quality. In Nepal, due to paucity of up to date regulations, there has been the risk of converting the whole country into a dumping ground for plethora of health related equipment and risk of providing compromised services.

Within a short span of just two decades, the numbers of medical colleges and higher institutions imparting health related education in Nepal has multiplied significantly. The numbers of human resources in these fields and especially in clinical biochemistry has multiplied too. Thus, there has been a need of support and conduct science research and facilitate the collaboration of interdisciplinary clinical and biomedical research and publish them and disseminate them in the right forum.

Our objective of launching the ACCLM is to encourage quality research publications targeting the local and regional scientists in particular. The journey has thus begun, but it has miles to go, uninterrupted towards sustainability and that requires continuous input from various stakeholders, the researchers, the companies involved in diagnostics, the service-vendors, planners and society in particular by conducting quality research and feeding it with quality research publication. Let there be drain from the brain than having the brain in the drain. 